$04 ; 13$

\title{
Ядерные тормозные способности изотопов водорода и гелия в бериллии, углероде и вольфраме
}

\author{
(C) А.Н. Зиновьев, П.Ю. Бабенко \\ Физико-технический институт им. А.Ф. Иофрфе РАН, Санкт-Петербург, Россия \\ ฯ E-mail: babenko@npd.ioffe.ru
}

Поступило в Редакцию 25 февраля 2020 г.

В окончательной редакции 11 июня 2020 г.

Принято к публикации 11 июня 2020 г.

\begin{abstract}
Рассчитаны ядерные тормозные способности изотопов водорода и гелия в материалах Ве, C, W, перспективных для использования в качестве первой стенки токамака реактора. Показано, что присутствие притягивающей ямы в потенциале значительно влияет на зависимость ядерных тормозных способностей от энергии соударения. Использование потенциалов, рассчитанных в приближении функционала плотности с притягивающей ямой, позволило получить более точные значения ядерных тормозных способностей для изотопов водорода, которые при малых энергиях отличаются на $27-60 \%$ от табличных данных. Результаты для разных изотопов водорода хорошо описываются универсальной кривой.
\end{abstract}

Ключевые слова: ядерные тормозные способности, изотопы водорода, гелий, бериллий, углерод, вольфрам, токамак-реактор.

DOI: 10.21883/PJTF.2020.18.49997.18265

Теоретические и экспериментальные работы по исследованию тормозных способностей атомных частиц в веществе в настоящее время ведутся широким фронтом (см., например, работы [1-6]) и входят в число приоритетных сообщений на международных конференциях по взаимодействию ионов с веществом [7-9]. Широкое международное сотрудничество по данной теме поощряется Международным агентством по атомной энергии (IAEA), которое регулярно публикует сборники новейших теоретических и экспериментальных достижений [10].

Для описания торможения частиц в веществе используется подход, развитый в работе [11], который различает потери энергии, связанные с упругим рассеянием налетающей частицы на ядрах атомов мишени (ядерные тормозные способности, ЯТС), и электронные тормозные способности (ЭТС), связанные с возбуждением и ионизацией электронной системы. Для легких частиц при энергиях выше нескольких $\mathrm{keV}$ доминируют ЭТС. $\mathrm{B}$ пристеночной плазме токамака, где энергии частиц составляют 10-200 eV, доминируют ЯТС. Понимание взаимодействия частиц плазмы с первой стенкой токамака является одной из основных проблем, которые необходимо решить для успешной работы токамакареактора.

В работе [12] для описания рассеяния частиц был предложен потенциал, получивший название ZBL, который используется в имеющем широкое распространение коде SRIM [13]. Из других известных потенциалов часто используются потенциалы Мольера [14] и Ленца-Йенсена [15]. В работе [16] было показано, что ЯТС, вычисленные с помощью потенциала ZBL, могут значительно отличаться от имевшихся в то время экспериментальных данных. Был предложен потенциал, получивший название $\mathrm{Kr}-\mathrm{C}$ [16], лучше описывающий эксперимент

В [17] были проанализированы данные о потенциалах, полученных из обработки эксперимента по рассеянию частиц, и предложен потенциал, наилучшим образом описывающий эксперимент. В работе [18] проводилось сравнение экспериментальных данных о потенциалах с расчетами, выполненными в приближении функционала плотности (DFT) с использованием пакета программ DMol для выбора базиса используемых волновых функций, и было достигнуто хорошее согласие с экспериментом (далее будем называть эти потенциалы DFT-потенциалами).

Ядерные тормозные способности удобно рассчитывать в системе центра масс (СЦМ), чтобы избежать многозначности зависимости угла рассеяния $\theta$ от параметра удара $p$, которая обычно имеет место при рассеянии на потенциалах с потенциальной ямой. Далее в настоящей работе будем использовать сечение тормозных потерь $S_{n}$, которое связано с тормозной способностью $d E / d R$ выражением

$$
S_{n}=\frac{d E}{d R} \frac{1}{N}=\int_{0}^{\infty} 2 \pi p T[\theta(p)] d p .
$$

Здесь $N$ - плотность мишени. Потеря энергии при соударении $T[\theta]$ выражается формулой

$$
T[\theta(p)]=\frac{4 M_{1} M_{2}}{\left(M_{1}+M_{2}\right)^{2}} \sin ^{2}\left[\frac{\theta(p)}{2}\right],
$$

$M_{1}$ и $M_{2}-$ массы сталкивающихся частиц, $\theta(p)-$ угол рассеяния в СЦМ,

$$
\theta(p)=\pi-2 p \int_{r_{0}}^{\infty} \frac{d r}{r^{2} \sqrt{1-\frac{U(r)}{E_{\mathrm{CM}}}-\frac{p^{2}}{r^{2}}}} .
$$



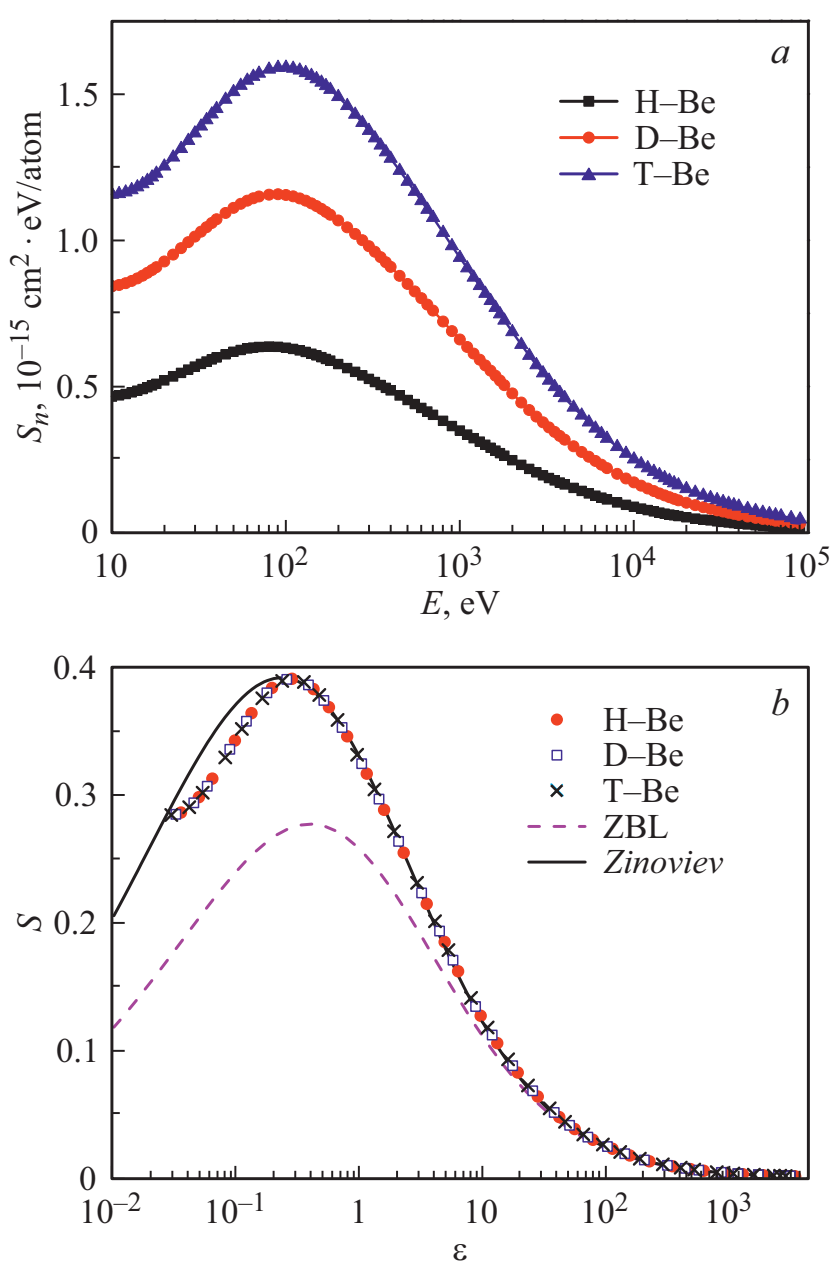

Рис. 1. $a-$ рассчитанные ядерные тормозные способности для изотопов водорода в бериллии. $b-$ сравнение вычисленных данных об ЯТС при столкновениях изотопов водорода с бериллием в универсальных координатах. Данные хорошо описываются единой кривой. Представлено сравнение с результатами, получаемыми при использовании потенциалов Зиновьева [17] и ZBL [12].

Здесь $U(r)$ п потенциал взаимодействия, $E_{\mathrm{CM}}=E M_{2} /\left(M_{1}+M_{2}\right)-$ энергия соударения в СЦМ, $r_{0}$ - корень уравнения

$$
1-\frac{U\left(r_{0}\right)}{E_{\mathrm{CM}}}-\frac{p^{2}}{r_{0}^{2}}=0 .
$$

Для описания рассеяния часто используются различные типы экранированных кулоновских потенциалов, которые могут быть записаны в виде

$$
U(r)=\frac{Z_{1} Z_{2} e^{2}}{r} \Phi\left(\frac{r}{a}\right),
$$

$Z_{1}$ и $Z_{2}$ - заряды ядер сталкивающихся частиц, $a-$ длина экранирования. В этом случае можно ввести универсальные координаты $\varepsilon=E / \varepsilon_{0}$ и $S=S_{n} / S_{0}$ (где $\left.\varepsilon_{0}=\left(Z_{1} Z_{2} e^{2} / a\right)\left(M_{1}+M_{2}\right) / M_{2}, S_{0}=\pi a^{2} \varepsilon_{0} 4 M_{1} M_{2} /\left(M_{1}+M_{2}\right)^{2}\right)$, предложенные в $[11,16]$. Для потенциалов, описываемых формулой (5), данные для различных систем описываются универсальной кривой.

Выбор длины экранирования $a$ зависит от типа потенциала. Для потенциала ZBL $a_{\mathrm{ZBL}}=0.8853 /\left(Z_{1}^{0.23}+Z_{2}^{0.23}\right)$. Используются атомные единицы. Для потенциала $\mathrm{Kr}-\mathrm{C}$ [16] и потенциала, предложенного в работе [17], применяется длина экранирования Фирсова [19] $a_{\mathrm{F}}=0.8853 /\left(Z_{1}^{2 / 3}+Z_{2}^{2 / 3}\right)^{1 / 2}$. Для потенциалов Мольера [14] и Ленца-Йенсена [15] используется длина экранирования, предложенная Линдхардом [11]: $a_{\mathrm{L}}=0.8853 /\left(Z_{1}^{1 / 2}+Z_{2}^{1 / 2}\right)^{2 / 3}$.

Как уже упоминалось, потенциалы взаимодействия, рассчитанные в DFT-приближении с использованием пакета программ DMol [18] для получения набора молекулярных волновых функций, дают лучшее согласие с экспериментом, чем ранее использовавшиеся потенциальные модели [12,14-16]. Расчеты потенциала для систем $\mathrm{H}-\mathrm{Be}, \mathrm{H}-\mathrm{C}, \mathrm{H}-\mathrm{W}$ [20], выполненные упомянутым выше методом, показали наличие ямы в потенциале, что приводит к притяжению частиц на больших расстояниях. Отталкивающая часть этого потенциала хорошо согласуется с формулой Зиновьева [17]. Отталкивающая часть потенциала ZBL уменьшается медленнее с увеличением межъядерных расстояний.

Глубину потенциальной ямы $U_{0}$ можно также определить по более точным данным об энергии диссоциации молекул. Для систем $\mathrm{C}-\mathrm{H}$ и $\mathrm{Be}-\mathrm{H} U_{0}=3.50 \pm 0.03$ и $2.340 \pm 0.022 \mathrm{eV}$ [21] соответственно. Положения минимумов в потенциале, рассчитанные методом DFT, совпали с данными спектроскопических измерений [22]. С использованием этих данных полученные значения потенциала для притягивающей части корректировались следующим образом: функциональная зависимость от межъядерного расстояния в области потенциальной ямы остается такой же, как было рассчитано, но масштаб энергии был пропорционально скорректирован для получения экспериментального значения глубины потенциальной ямы $U_{0}$.

Потенциалы взаимодействия для различных изотопов водорода практически совпадают. Различие в массах сталкивающихся частиц влияет только на величину приведенной массы электрона, а кулоновское взаимодействие электрона с ядрами остается тем же самым. Глубина потенциальной ямы $U_{0}$ и положение минимума для систем C-H и C-D отличаются в третьем знаке $[21,22]$. Аналогичная ситуация для систем $\mathrm{H}-\mathrm{W}, \mathrm{D}-\mathrm{W}, \mathrm{T}-\mathrm{W}$ и $\mathrm{H}-\mathrm{Be}, \mathrm{D}-\mathrm{Be}, \mathrm{T}-\mathrm{Be}$. Рекомендуемые значения потенциалов для рассматриваемых систем опубликованы в работе [20].

Расчеты проводились для диапазона начальных энергий $E=10-10^{6} \mathrm{eV}$. При энергии $10 \mathrm{eV}$ длина волны де Бройля $\lambda=0.09 \AA$. Характерная длина изменения потенциала при этой энергии определяется параметрами потенциальной ямы и составляет $1-1.5 \AA$ для рассматриваемых систем. В получаемую величину $S_{n}$ вносят основной вклад параметры удара, для которых $\theta>p / \lambda$. Вклад малых углов рассеяния, где это условие 
нарушается, составляет менее $2 \%$. При меньших энергиях необходимо квантовое рассмотрение рассеяния, поскольку понятие траектории исчезает.

Рассчитанные ядерные тормозные способности для изотопов водорода в Ве представлены на рис. 1, $a$. Различие в тормозных способностях для разных изотопов обусловлено пропорциональностью фактора $T[\theta(p)] \sim M_{1} M_{2} /\left(M_{1}+M_{2}\right)^{2}$. Как и следовало ожидать, в универсальных координатах кривые для различных изотопов совпадают (см. рис. $1, b$ ). На рис. $1, b$ приведено сравнение полученных данных с результатами, получаемыми при использовании потенциала Зиновьева и ZBL. Как видно из рисунка, при малых энергиях $\varepsilon<1$ отличие полученных результатов от табличных данных кода SRIM, где используется потенциал ZBL, достигает $27-60 \%$, т. е. весьма существенно. Согласие с расчетами с использованием потенциала Зиновьева заметно лучше. Наличие притягивающей ямы в потенциале обусловливает отклонение от универсальной кривой при малых $\varepsilon$. При $\varepsilon>10$ предсказания различных теоретических моделей совпадают.

На рис. 2 представлено сопоставление полученных данных для систем $(\mathrm{H}, \mathrm{D}, \mathrm{T})-\mathrm{Be}, \mathrm{C}, \mathrm{W}$. Данные для разных изотопов совпадают. Данные для потенциала ZBL приведены для системы Н-C, где длины экранирования Фирсова и ZBL практически одинаковы. Как видно из рисунка, табличные данные кода SRIM значительно занижают ЯТС. Наличие ямы в потенциале проявляется во всех случаях в виде отклонений от кривой, рассчитанной с использованием потенциала без притягивающей ямы. Смещение положений перегиба кривой при малых значениях $\varepsilon$ в универсальных координатах связано с фактором $\varepsilon_{0}$. Для рассматриваемых систем параметр $\varepsilon_{0}$ составляет $284 \mathrm{eV}$ для случая $\mathrm{H}-\mathrm{Be}, 456 \mathrm{eV}$ для случая $\mathrm{H}-\mathrm{C}$ и $10334 \mathrm{eV}$ для случая $\mathrm{H}-\mathrm{W}$. Отклонение прояв-

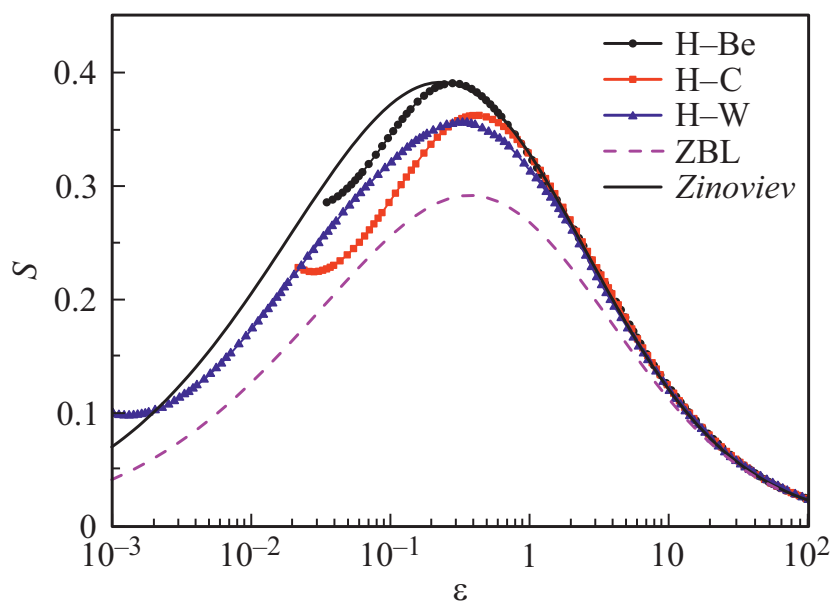

Pис. 2. ЯТС для столкновений изотопов водорода с мишенями из бериллия, углерода и вольфрама. В универсальных координатах данные для различных изотопов совпадают, но зависят от материала мишени. Приведено сопоставление с результатами расчетов при использовании потенциалов Зиновьева [17] и ZBL [12].

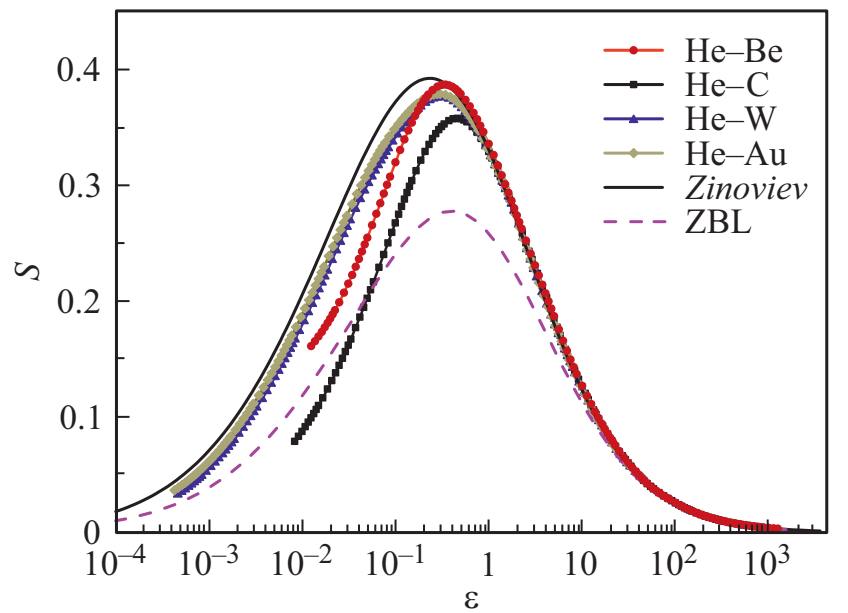

Рис. 3. ЯТС для столкновений атомов гелия с Be, C, W и $\mathrm{Au}$ в универсальных координатах. Представлены также расчеты с использованием потенциалов Зиновьева [17] и ZBL [12].

ляется при значениях $\varepsilon=U_{0} / \varepsilon_{0} \sim 1$, где $U_{0}-$ глубина ямы.

На рис. 3 представлены ядерные тормозные способности для атомов гелия. В этом случае притягивающая яма в потенциале практически отсутствует. Для тяжелых элементов имеется хорошее согласие с результатами расчета с использованием потенциала [17]. Данные, полученные с использованием DFT-потенциала, дают более точный результат. Как и для изотопов водорода, в случае атомов Не табличные данные кода SRIM значительно занижают ЯТС при малых энергиях.

Таким образом, рассчитаны ЯТС для изотопов водорода и гелия при соударениях с $\mathrm{Be}, \mathrm{C}, \mathrm{W}$ - материалами, перспективными для использования в качестве первой стенки токамака-реактора.

Использование DFT-потенциалов с коррекцией параметров потенциальной ямы, основанной на данных спектроскопических измерений, позволило получить более точные результаты, которые отличаются на 27-60\% от табличных данных. Показано влияние потенциальной ямы на ЯТС при малых энергиях соударения. Данные для различных изотопов водорода хорошо описываются универсальной кривой.

\section{Финансирование работы}

Работа выполнена в рамках государственного задания Министерства образования и науки РФ для Федерального государственного бюджетного учреждения науки Физико-технического института им. А.Ф. Иоффе Российской академии наук.

\section{Конфликт интересов}

Авторы заявляют, что у них нет конфликта интересов. 


\section{Список литературы}

[1] Sigmund P., Schinner A. // J. Appl. Phys. 2020. V. 127. N 16. P. 164302. DOI: $10.1063 / 5.0005292127$

[2] Montanari C.C., Miraglia J.E. // Phys. Rev. A. 2017. V. 96. N 1. P. 012707. DOI: 10.1103/PhysRevA.96.012707

[3] Paul H., Sanchez-Parcerisa D. // Nucl. Instrum. Meth. Phys. Res. B. 2013. V. 312. P. 110-117. DOI: 10.1016/j.nimb.2013.07.012

[4] Jedrejcic D., Greife U. // Nucl. Instrum. Meth. Phys. Res. B. 2018. V. 428. P. 1-8. DOI: 10.1016/j.nimb.2018.04.039

[5] Naqvi S.R., Possnert G., Primetzhofer D. // Nucl. Instrum. Meth. Phys. Res. B. 2016. V. 371. P. 76-80.

DOI: 10.1016/j.nimb.2015.09.048

[6] Parfitt W.A., Jackman R.B. // Nucl. Instrum. Meth. Phys. Res. B. 2020. V. 478. P. 21-23. DOI: 10.1016/j.nimb.2020.05.015

[7] Paul H. // AIP Conf. Proc. 2013. V. 1525. P. 309-313. DOI: $10.1063 / 1.4802339$

[8] Montanari C. Stopping power, experimental trends and open subjects, with focus on the low to intermediate energy region // Abstract book of the 28 Int. Conf. on atomic collisions in solids (ICACS-28). Caen, France, 2018. INV02-119.

[9] Sigmund P., Kuzmin V., Schinner A. Reciprocity analysis of electronic stopping of slow ions // Abstract book of the 28 Int. Conf. on atomic collisions in solids (ICACS-28). Caen, France, 2018. TO1.2-O1-82.

[10] Improvement of the reliability and accuracy of heavy ion beam analysys. Thechnical reports series. N 485 / Ed. A. Simon. Vienna: IAEA, 2019. 198 p.

[11] Linchard J., Nielsen V., Scharff M. // Mat. Fys. Medd. Dan. Vid. Selsk. 1968. V. 36. N 10. P. 1-32.

[12] Ziegler J.F., Biersack J.P., Littmark U. The stopping and range of ions in solids. Ser. Stopping and range of ions in matter. V. 1. N.Y.: Pergamon, 1985. 321 p.

[13] Ziegler J.F., Biersack J.P. SRIM [Электронный pecypc]. Режим доступа: http://www.srim.org

[14] Moliere G. // Z. Naturforsch. A. 1947. V. 2. N 3. P. 133-145. DOI: 10.1515/zna-1947-0302

[15] Jensen H.Z. // Z. Phys. 1932. V. 77. N 11-12. P. 722-745. DOI: $10.1007 / \mathrm{BF} 01342151$

[16] Wilson W.D., Haggmark L.G., Biersack J.P. // Phys. Rev. B. 1977. V. 15. N 5. P. 2458-2468. DOI: $10.1103 /$ PhysRevB.15.2458

[17] Зиновьев А.Н. // ЖТФ. 2008. Т. 78. В. 1. С. 15-20. [Пер. версия: 10.1134/S1063784208010039].

[18] Zinoviev A.N., Nordlund K. // Nucl. Inst. Meth. Phys. Res. B. 2017. V. 406. P. 511-517. DOI: 10.1016/j.nimb.2017.03.047

[19] Фирсов О.Б. // ЖЭТФ. 1958. Т. 34. В. 2. С. 447-452.

[20] Meluzova D.S., Babenko P.Yu., Shergin A.P., Nordlund K., Zinoviev A.N. // Nucl. Instrum. Meth. Phys. Res. B. 2019. V. 460. P. 4-9. DOI: 10.1016/j.nimb.2019.03.037

[21] Darwent D. Bond dissociation energies in simple molecules. Catholic University of America, 1970. 60 p. NSRDS-NBS.31.

[22] Никольский Б.П. Справочник химика. Т. 1. Л.: Химия, 1966. $1072 \mathrm{c}$. 